\title{
Generic diffeomorphisms with weak limit shadowing
}

\author{
Gang Lu' ${ }^{1}$ Keonhee Lee ${ }^{2}$ and Manseob Lee ${ }^{3 *}$
}

"Correspondence:

Imsds@mokwon.ac.kr

${ }^{3}$ Department of Mathematics,

Mokwon University, Daejeon,

302-729, Korea

Full list of author information is

available at the end of the article

\begin{abstract}
In this paper, we show that if a $C^{1}$-generic diffeomorphism has the weak limit shadowing property on the chain recurrent set, then the diffemorphism satisfies Axiom A and the no-cycle condition.
\end{abstract}

MSC: $37 C 50 ; 37 D 30$

Keywords: shadowing; weak limit shadowing; $\Omega$-stable; generic

\section{Introduction}

Let $M$ be a closed $C^{\infty}$ manifold, and let $\operatorname{Diff}(M)$ be the space of diffeomorphisms of $M$ endowed with the $C^{1}$-topology. Denote by $d$ the distance on $M$ induced from a Riemannian metric $\|\cdot\|$ on the tangent bundle TM. Let $f \in \operatorname{Diff}(M)$ and $\Lambda$ be a closed $f$-invariant set. For $\delta>0$, a sequence of points $\left\{x_{i}\right\}_{i=a}^{b}(-\infty \leq a<b \leq \infty)$ in $M$ is called a $\delta$-pseudo orbit of $f$ if $d\left(f\left(x_{i}\right), x_{i+1}\right)<\delta$ for all $a \leq i \leq b-1$.

We say that $f$ has the shadowing property on $\Lambda$ if for every $\epsilon>0$, there is $\delta>0$ such that for any $\delta$-pseudo orbit $\left\{x_{i}\right\}_{i=a}^{b} \subset \Lambda$ of $f(-\infty \leq a<b \leq \infty)$, there is a point $y \in M$ such that $d\left(f^{i}(y), x_{i}\right)<\epsilon$ for all $a \leq i \leq b-1$. In the dynamical systems, the shadowing theory is a very useful notion. In fact, it deals with the stability theorem (see [1]). For instance, Robinson [2] proved that if a diffeomorphism $f$ is structurally stable, then it has the shadowing property. In [3] Sakai showed that $f$ belongs to the $C^{1}$-interior of the shadowing property if and only if $f$ is structurally stable. In this paper, we deal with another shadowing property, that is, the weak limit shadowing property which was studied by [4].

We say that $f$ has the weak limit shadowing property on $\Lambda$ (or $\Lambda$ is weak limit shadowable for $f$ ) if there exists a $\delta>0$ with the following property: if a sequence $\left\{x_{i}\right\}_{i \in \mathbb{Z}} \subset \Lambda$ is a $\delta$ pseudo orbit of $f$, for which relations $d\left(f\left(x_{i}\right), x_{i+1}\right) \rightarrow 0$ as $i \rightarrow+\infty$ and $d\left(f^{-1}\left(x_{i+1}\right), x_{i}\right) \rightarrow 0$ as $i \rightarrow-\infty$ hold, then there is a point $y \in M$ such that $d\left(f^{i}(y), x_{i}\right) \rightarrow 0$ as $i \rightarrow \pm \infty$. Note that if $f$ has the limit shadowing property, then $f$ has the weak limit shadowing property. But the converse is not true (see [4, Example 4]). Denote by $P(f)$ the set of periodic points of $f$. Then $P(f) \subset \Omega(f) \subset \mathcal{R}(f)$, where $\Omega(f)$ is the set of non-wandering points of $f$, and $\mathcal{R}(f)$ is the set of chain recurrent points of $f$. Note that if $f$ satisfies Axiom A and the nocycle condition, then $\Omega(f)=\mathcal{R}(f)$. We say that $f$ has the s-limit shadowing property on $\Lambda$ if for any $\epsilon>0$, there is a $\delta>0$ such that for any $\delta$-limit pseudo orbit $\xi=\left\{x_{i}\right\}_{i \in \mathbb{Z}} \subset \Lambda$, there is a point $y \in M$ such that $d\left(f^{i}(y), x_{i}\right)<\epsilon$ for all $i \in \mathbb{Z}$, and $d\left(f^{i}(y), x_{i}\right) \rightarrow 0$ as $i \rightarrow \pm \infty$. Clearly, the weak limit shadowing property is a weak notion of the $s$-limit shadowing property. We say that $\Lambda$ is hyperbolic if the tangent bundle $T_{\Lambda} M$ has a $D f$-invariant splitting $E^{s} \oplus E^{u}$ 
and there exist constants $C>0$ and $0<\lambda<1$ such that

$$
\left\|\left.D_{x} f^{n}\right|_{E_{x}^{s}} ^{s}\right\| \leq C \lambda^{n} \quad \text { and } \quad\left\|\left.D_{x} f^{-n}\right|_{E_{x}^{u}}\right\| \leq C \lambda^{n}
$$

for all $x \in \Lambda$ and $n \geq 0$. If $\Lambda=M$, then $f$ is Anosov. Very recently, Sakai [5] showed that if a $C^{1}$-generic diffeomorphism $f$ has the $s$-limit shadowing property on $\mathcal{R}(f)$, then $f$ satisfies Axiom A and the no-cycle condition. The result is motivation for this study. The main theorem of the paper is as follows.

Theorem 1.1 For $C^{1}$-generic $f$, iff has the weak limit shadowing property on $\mathcal{R}(f)$, then $f$ satisfies Axiom A and the no-cycle condition.

\section{Proof of Theorem 1.1}

Let $M$ be as before and $f \in \operatorname{Diff}(M)$. Let $p \in P(f)$ be a hyperbolic saddle with period $\pi(p)>0$. The stable manifold $W^{s}(p)$ and the unstable manifold $W^{u}(p)$ are defined as follows. It is well known that if $p$ is a hyperbolic periodic point of $f$ with a period $k$, then the sets

$$
\begin{aligned}
& W^{s}(p)=\left\{x \in M: f^{k n}(x) \rightarrow p \text { as } n \rightarrow \infty\right\} \text { and } \\
& W^{u}(p)=\left\{x \in M: f^{-k n}(x) \rightarrow p \text { as } n \rightarrow \infty\right\}
\end{aligned}
$$

are $C^{1}$-injectively immersed submanifolds of $M$. Let $p, q \in P(f)$ be saddles. Let $P(f)$ be the set of periodic points of $f$. Denote by $\mathcal{O}_{f}(p)$ the periodic $f$-orbit of $p \in P(f)$. We denote $p \sim q$ if the intersections $W^{s}\left(\mathcal{O}_{f}(p)\right) \pitchfork W^{u}\left(\mathcal{O}_{f}(q)\right) \neq \emptyset$ and $W^{u}\left(\mathcal{O}_{f}(p)\right) \pitchfork W^{s}\left(\mathcal{O}_{f}(q)\right) \neq \emptyset$. Then we know that if $p \sim q$, then $\operatorname{index}(p)=\operatorname{index}(q)$. Here index $(p)$ is the dimension of the stable manifold of $p$, that is, $\operatorname{dim} W^{s}(p)$.

Proposition 2.1 There is a residual set $\mathcal{G}_{1} \subset \operatorname{Diff}(M)$ such that for any $f \in \mathcal{G}$, iff $\left.\right|_{\mathcal{R}(f)}$ has the weak limit shadowing property, then for any saddles $p, q \in P(f)$, index $(p)=\operatorname{index}(q)$.

To prove Proposition 2.1, we need the following lemma.

Lemma 2.2 Let $p, q \in P(f)$ be saddles. If $f$ has the weak limit shadowing property on $\mathcal{R}(f)$, then $W^{s}\left(\mathcal{O}_{f}(p)\right) \cap W^{u}\left(\mathcal{O}_{f}(q)\right) \neq \emptyset$.

Proof Suppose that $f$ has the weak limit shadowing property on $\mathcal{R}(f)$. For any saddles $p, q \in P(f)$, we show that $W^{u}\left(\mathcal{O}_{f}(p)\right) \cap W^{s}\left(\mathcal{O}_{f}(q)\right) \neq \emptyset$. For the sake of simplicity, we may assume that $f(p)=p$ and $f(q)=q$. Let $\delta>0$ be the number of the weak limit shadowing property of $f$ such that $d(p, q)<\delta$. We construct $\delta$-limit pseudo orbit $\xi=\left\{x_{i}\right\}_{i \in \mathbb{Z}} \subset \mathcal{R}(f)$ as follows. (i) $x_{0}=p$, (ii) $x_{-i}=f^{-i}(p)$ for all $i>0$, (iii) $x_{i}=f^{i}(q)$ for all $i \geq 1$. Then the $\delta$-limit pseudo orbit

$$
\xi=\left\{\ldots, x_{-1}, p=\left(x_{0}\right), q\left(=x_{1}\right), x_{2}, \ldots\right\}=\{\ldots, p, p, q, q, \ldots\}
$$

and it is clear that $\xi \subset \mathcal{R}(f)$. Since $f$ has the weak shadowing property on $\mathcal{R}(f)$, there is a point $y \in M$ such that $d\left(f^{i}(y), x_{i}\right) \rightarrow 0$ as $i \rightarrow \pm \infty$. Then $f^{i}(y) \rightarrow p$ as $i \rightarrow-\infty$ and $f^{1+i}(y) \rightarrow q$ as $i \rightarrow \infty$. Hence, $y \in W^{s}(p)$ and $f(y) \in W^{s}(q)$. Thus, $W^{u}(p) \cap W^{s}(q) \neq \emptyset$. 
The following is called the Kupka-Smale theorem.

Lemma 2.3 There is a residual set $\mathcal{G}_{1} \subset \operatorname{Diff}(M)$ such that for any $f \in \mathcal{G}$, every periodic point is hyperbolic, and the stable manifolds and the unstable manifolds of periodic points are all transverse.

Proof of Proposition 2.1 Let $f \in \mathcal{G}_{1}$, and let $p, q \in P(f)$ be saddles. Suppose that $f$ has the weak limit shadowing property on $\mathcal{R}(f)$. Let $\delta>0$ be the number of the weak limit shadowing property of $f$ such that $d(p, q)<\delta$. Then we will drive a contradiction, we may assume that $\operatorname{index}(p) \neq \operatorname{index}(q)$. Then we know that $\operatorname{dim} W^{s}(p)+\operatorname{dim} W^{u}(q)<\operatorname{dim} M$ or $\operatorname{dim} W^{u}(p)+\operatorname{dim} W^{s}(q)<\operatorname{dim} M$. In this proof, we consider that $\operatorname{dim} W^{s}(p)+\operatorname{dim} W^{u}(q)<$ $\operatorname{dim} M$ (the other case is similar). Since $f \in \mathcal{G}_{1}, W^{s}(p) \cap W^{u}(q)=\emptyset$. Since $f$ has the weak limit shadowing property on $\mathcal{R}(f)$, by Lemma $2.2, W^{s}(p) \cap W^{u}(q) \neq \emptyset$. This is a contradiction.

Let $p \in P(f)$ be a hyperbolic saddle with a period $\pi(p)>0$. Then there are the local stable manifold $W_{\epsilon}^{s}(p)$ and the unstable manifold $W_{\epsilon}^{u}(p)$ of $p$ for some $\epsilon=\epsilon(p)>0$. It is easily seen that if $d\left(f^{n}(x), f^{n}(p)\right) \leq \epsilon$ for all $n \geq 0$, then $x \in W_{\epsilon}^{s}(p)$, and if $d\left(f^{n}(x), f^{n}(p)\right) \leq \epsilon$ for all $n \leq 0$, then $x \in W_{\epsilon}^{u}(p)$. The following lemma shows that if $f$ has the $s$-limit shadowing property on $\mathcal{R}(f)$, then the numbers of sinks and sources are finite (see [5, Lemma 2]). From the above facts, we show that if $f$ has the weak limit shadowing property on $\mathcal{R}(f)$, then the numbers of sinks and sources are finite.

Lemma 2.4 Let $f$ have the weak limit shadowing property on $\mathcal{R}(f)$, and let $\delta>0$ be the number of the weak limit shadowing property of $f$. For any saddle $q \in P(f)$, if $p \in P(f)$ is a sink or a source, then $d(p, q) \geq \delta$.

Proof We will derive a contradiction. Suppose that $q \in P(f)$ is a saddle and $p \in P(f)$ is a sink with $d(p, q)<\delta$. For the sake of simplicity, we may assume that $f(p)=p, f(q)=q$. Since $q$ is a saddle, there is $\epsilon(q)>0$ such that if for any $x \in M, d\left(f^{i}(x), f^{i}(q)\right) \leq \epsilon(q)$ as $i \rightarrow \infty$, then $x \in W_{\epsilon(q)}^{s}(q)$, and if $x \in M, d\left(f^{i}(x), f^{i}(q)\right) \leq \epsilon(q)$ as $i \rightarrow-\infty$, then $x \in W_{\epsilon(q)}^{u}(q)$. Then we may assume that $d(p, q)>\epsilon(q)$. Then we construct a $\delta$-limit pseudo orbit $\xi=\left\{x_{i}\right\}_{i \in \mathbb{Z}} \subset \mathcal{R}(f)$ as follows. Put $x_{-i}=f^{-i}(p)$ for $i \geq 0$ and $x_{i}=f^{i}(q)$ for $i \geq 1$. Then $\xi=\left\{x_{i}\right\}_{i \in \mathbb{Z}}$ is clearly a $\delta$-limit pseudo orbit of $f$, and $\xi=\left\{x_{i}\right\}_{i \in \mathbb{Z}} \subset \mathcal{R}(f)$. Since $f$ has the weak limit shadowing property on $\mathcal{R}(f)$, there is a point $y \in M$ such that $d\left(f^{i}(y), x_{i}\right) \rightarrow 0$ as $i \rightarrow \pm \infty$. Since $p$ is a sink, $d\left(f^{-i}(y), x_{-i}\right)=d\left(f^{-i}(y), p\right) \rightarrow 0$ as $i \rightarrow \infty$. Then $y=p$. Since $d\left(f^{i}(y), x_{i}\right)=d\left(f^{i}(y), q\right) \rightarrow 0$ as $i \rightarrow \infty$, there is $k>0$ such that $d\left(f^{k+i}(y), f^{k+i}(q)\right)=d\left(f^{k+i}(y), q\right) \leq \epsilon(q)$ for $i \geq 0$. Then $f^{k}(y) \in W_{\epsilon(q)}^{s}(q)$. Since $y=p$, we know that $d(p, q) \leq \epsilon(q)$. This is a contradiction.

Let $p$ be a periodic point of $f$, and let $0<\delta<1$. We say $p$ has a $\delta$-weak eigenvalue provided $D_{p} f^{\pi(p)}$ has an eigenvalue $\lambda$ such that $(1-\delta)^{\pi(p)}<|\lambda|<(1+\delta)^{\pi(p)}$. We say that the periodic point has a real spectrum if all of its eigenvalues are real and a simple spectrum if all of its eigenvalues have multiplicity one. The following lemma will play a crucial role in our proof.

Lemma 2.5 [6, Lemma 5.1] There is a residual set $\mathcal{G}_{2} \subset \operatorname{Diff}(M)$ such that for any $f \in \mathcal{G}_{2}$, 
(a) for any $\eta>0$, if for any $C^{1}$-neighborhood $\mathcal{U}(f)$ off, there exist $g \in \mathcal{U}(f)$ and $p_{g}, q_{g} \in P(g)$ with the same period such that $d\left(p_{g}, q_{g}\right)<\eta$, then there exist $p, q \in P(f)$ with the same period such that $d(p, q)<\eta$;

(b) for any $\eta>0$, if for any $C^{1}$-neighborhood $\mathcal{U}(f)$ off, there exist $g \in \mathcal{U}(f)$ and $p_{g} \in P(g)$ with an $\eta$-weak eigenvalue, then there exist $p \in P(f)$ with a $2 \eta$-weak eigenvalue;

(c) for any $\eta>0$, if $q \in P(f)$ with an $\eta$-weak eigenvalue and a real spectrum, then there exists $p \in P(f)$ with an $\eta$-weak eigenvalue with a simple real spectrum.

Lemma 2.6 [7, Lemma 5.1] There is a residual set $\mathcal{G}_{3} \subset \operatorname{Diff}(M)$ such that for any $f \in \mathcal{G}_{3}$, for any $\eta>0$, iffor any $C^{1}$-neighborhood $\mathcal{U}(f)$ off, there exist $g \in \mathcal{U}(f)$ and $p_{g}, q_{g} \in P(g)$ with the same period such that $d\left(p_{g}, q_{g}\right)<\eta$ with different indices, then there exist $p, q \in P(f)$ with the same period such that $d(p, q)<\eta$ with different indices.

The following so-called Franks lemma will play an essential role in our proof.

Lemma 2.7 Let $\mathcal{U}(f)$ be any given $C^{1}$-neighborhood of $f$. Then there exists $\epsilon>0$ and a $C^{1}$-neighborhood $\mathcal{U}_{0}(f) \subset \mathcal{U}(f)$ off such that for given $g \in \mathcal{U}_{0}(f)$, a finite set $\left\{x_{1}, x_{2}, \ldots, x_{N}\right\}$, a neighborhood $U$ of $\left\{x_{1}, x_{2}, \ldots, x_{N}\right\}$ and linear maps $L_{i}: T_{x_{i}} M \rightarrow T_{g\left(x_{i}\right)} M$ satisfying $\| L_{i}-$ $D_{x_{i}} g \| \leq \epsilon$ for all $1 \leq i \leq N$, there exists $g^{\prime} \in \mathcal{U}(f)$ such that $g^{\prime}(x)=g(x)$ if $x \in\left\{x_{1}, x_{2}, \ldots, x_{N}\right\} \cup$ $(M \backslash U)$ and $D_{x_{i}} g^{\prime}=L_{i}$ for all $1 \leq i \leq N$.

If $p \in P(f)$ is hyperbolic, then for any $g \in \mathcal{U}(f)$, there is a unique hyperbolic periodic point $p_{g} \in P(g)$ nearby $p$ such that $\pi\left(p_{g}\right)=\pi(p)$ and index $\left(p_{g}\right)=\operatorname{index}(p)$, where index $=$ $\operatorname{dim} W^{s}(p)$. Such a $p_{g}$ is called the continuation of $p$.

Lemma 2.8 There is a residual set $\mathcal{G}_{4} \subset \operatorname{Diff}(M)$ such that for any $f \in \mathcal{G}_{4}$, iff has the weak limit shadowing property on $\mathcal{R}(f)$, then there is $\eta>0$ such that $f$ has no $\eta$-weak eigenvalue.

Proof Let $f \in \mathcal{G}_{4}=\mathcal{G}_{2} \cap \mathcal{G}_{3}$. To derive a contradiction, we may assume that for any $\eta>0$, there is a hyperbolic periodic point $q_{g}$ of $g\left(C^{1}\right.$-nearby $\left.f\right)$ such that $q_{g}$ has an $\eta$-weak eigenvalue and a simple real spectrum. Let $\delta>0$ be the number of the weak limit shadowing property of $f$ such that $0<\eta<\delta / 2$. For the sake of simplicity, we assume that $q_{g}$ is a fixed point. By Lemma 2.7, there is $h C^{1}$-close to $g$ and $h C^{1}$-nearby $f$ such that $q_{h}$ has 1 as an eigenvalue. By Lemma 2.7 and as in the proof of [8, Lemma 2.4], we can construct an $h^{l}$ $(l>0)$-invariant small arc $\mathcal{I}_{q_{h}}$ of $h^{l}$ containing $q_{h}$ such that $d\left(p_{h}, r_{h}\right)<\eta$, where $p_{h}, r_{h}$ are the end points of $\mathcal{I}_{q h}, h^{l}\left(p_{h}\right)=p_{h}, h^{l}\left(r_{h}\right)=r_{h}$ and $p_{h}, r_{h}$ are hyperbolic saddles and different indices. Since $f \in \mathcal{G}_{4}$, there exist $p, r \in P(f)$ with the same period such that $d(p, r)<\eta$ with different indices. Since $f$ has the weak limit shadowing property on $\mathcal{R}(f)$, and by Lemma 2.4, $p, r \in P(f)$ are saddles. Since $d(p, r)<\delta$, by Proposition 2.1, we know that $\operatorname{index}(p)=\operatorname{index}(r)$. But, since index $(p) \neq \operatorname{index}(r)$, this is a contradiction.

Denote by $\mathcal{F}(M)$ the $C^{1}$-interior of the set of diffeomorphisms of $M$ whose periodic points are all hyperbolic. In [9], Hayashi showed that if $f \in \mathcal{F}(M)$, then $f$ satisfies Axiom A and the no-cycle condition. To prove Theorem 1.1, it is enough to show $f \in \mathcal{F}(M)$.

End of proof of Theorem 1.1 Let $f \in \mathcal{G}_{4}$, and let $f$ have the weak limit shadowing property on $\mathcal{R}(f)$. If not, then $f \notin \mathcal{F}(M)$. There is $g C^{1}$-closed to $f$ and a non-hyperbolic periodic 
point $p_{g}$ such that the point $p_{g}$ has an $\eta / 2$ weak eigenvalue. Since $f \in \mathcal{G}_{4}$, there is $p \in P(f)$ such that $p$ has an $\eta$-weak eigenvalue. By Lemma 2.8 , this is a contradiction.

\section{Competing interests}

The authors declare that they have no competing interests.

\section{Authors' contributions}

All authors conceived of the study, participated in its design and coordination, drafted the manuscript, participated in the sequence alignment, and read and approved the final manuscript.

\section{Author details}

${ }^{1}$ Department of Mathematics, School of Science, Shenyang University of Technology, Shenyang, 110178, P.R. China. ${ }^{2}$ Department of Mathematics, Chungnam National University, Daejeon, 305-764, Korea. ${ }^{3}$ Department of Mathematics, Mokwon University, Daejeon, 302-729, Korea.

\section{Acknowledgements}

We wish to thank the referee for carefully reading of the manuscript and for providing us with many good suggestions. GL is supported by the Doctoral Science Foundation of Liaoning Province by Hall of Liaoning province science and technology (No. 2012-1055). KL is supported by the National Research Foundation (NRF) of Korea funded by the Korean Government (No. 2011-0015193). ML is supported by the Basic Science Research Program through the National Research Foundation of Korea (NRF) funded by the Ministry of Education, Science and Technology, Korea (No. 2011-0007649).

Received: 1 November 2012 Accepted: 11 January 2013 Published: 4 February 2013

\section{References}

1. Pilyugin, SY: Shadowing in Dynamical Systems. Lecture Notes in Math. vol. 1706. Springer, Berlin (1999)

2. Robinson, C: Stability theorems and hyperbolicity in dynamical systems. Rocky Mt. J. Math. 7, 425-437 (1977)

3. Sakai, K: Pseudo orbit tracing property and strong transversality of diffeomorphisms on closed manifolds. Osaka J. Math. 31, 373-386 (1994)

4. Lee, K: Hyperbolic sets with the strong limit shadowing property. J. Inequal. Appl. 6, 507-517 (2001)

5. Sakai, K: Diffeomorphisms with s-limit shadowing property. Dyn. Syst. 27, 403-410 (2012)

6. Arbieto, A: Periodic orbits and expansiveness. Math. Z. 269, 801-807 (2011)

7. Arbieto, A, Senos, L, Sodero, T: The specification property for flows from the robust and generic viewpoint. J. Differ. Equ. 253, 1893-1909 (2012)

8. Sakai, K, Sumi, N, Yamamoto, K: Diffeomorphisms satisfying the specification property. Proc. Am. Math. Soc. 138, 315-321 (2010)

9. Hayashi, S: Diffeomorphisms in $\mathcal{F}^{1}(M)$ satisfy Axiom A. Ergod. Theory Dyn. Syst. 12, 233-253 (1992)

doi:10.1186/1687-1847-2013-27

Cite this article as: Lu et al.: Generic diffeomorphisms with weak limit shadowing. Advances in Difference Equations 2013 2013:27.

\section{Submit your manuscript to a SpringerOpen ${ }^{\ominus}$ journal and benefit from:}

- Convenient online submission

- Rigorous peer review

- Immediate publication on acceptance

- Open access: articles freely available online

- High visibility within the field

- Retaining the copyright to your article 